\title{
Peculiari caratteristiche delle oscillazioni libere del lago di Bolsena $\left({ }^{*}\right)$
}

\author{
P. CALOI
}

Ricevuto il 15 Aprile 1965

Riassunto. - Dall'esame di alcune serie di registrazioni limnografiche, ottenute sul lago di Bolsena, risulta che - generalmente - si formano, in detto lago, due serie di uninodali, di periodi e di ampiezze lievemente diversi. Quando le ampiezze pressoché coincidono, insorgono lunghe serie di battimenti caratteristici. Si determinano i periodi propri delle uninodali componenti.

Le uninodali, di ampiezza notevolmente superiore a quella di similari laghi italiani, determinano a Marta sensibili variazioni della verticale ap. parente, registrate con clinografi.

I e ampiezze delle uninodali a Marta sono, di consueto, circa 3 volte maggiori di quelle contemporaneamente registrate a Bolsena, nell'estremo Nord.

Summary. - Some series of limnographic records, obtained in the Bolsena Lake, show, generally, two series of uninodals with periods and amplitudes slightly different. When the amplitudes are about identical, there are long series of characteristic beats. The proper periods of the uninodal components are determined.

The uninodals - with amplitude a lot larger than those of the similar Italian Lakes - cause in Marta sensible variations of the apparent vertical with clinographs recorded.

The amplitudes of the uninodals are in Marta, usually three times about larger than these at the same time in the Nothern side of the Bolsena Lake, recorded.

1. - Le prime indagini sulle "sesse" del lago di Bolsena furono eseguite da L. Palazzo (1). Furono limitate ad alcune registrazioni, ef-

(*) Questo lavoro è stato condotto con contributi del Consiglio Nazionale delle Ricerche. 
fettuate a Marta e a Bolsena con un apparecchio "Sarasin ». Esse rivelarono l'esistenza, pressoché continua, di ampie oscillazioni libere, particolarmente sensibili a Marta, dove potevano raggiungere ampiezze cospicue, talvolta fra i 15 e i $20 \mathrm{~cm}$.

Più recentemente, nel quadro del vasto programma di ricerche teorico-sperimentali da me proposto ed iniziato nel $1948\left(^{2}\right)$, D. Di Filippo ebbe a studiare con vari metodi le oscillazioni stazionarie del bacino del lago di Bolsena $\left({ }^{3}, 4\right)$. Fra i risultati di questa indagine, veniva accertato che il periodo dell'oscillazione fondamentale (uninodale) del lago di Bolsena era di $13^{\mathrm{m}} .1$ circa.

2. - Alcuni anni or sono, mi proposi di ottenere una più sistematica serie di registrazioni delle sesse del lago di Bolsena, che - specie dopo le osservazioni eseguite su parecehi altri laghi (Garda, Maggiore, Bracciano, Levico, Caldonazzo, Scanno, Albano, ...) - apparivano fra le più ampie (se non le più ampie, in senso assoluto) dei laghi italiani, compresi i maggiori.

A tale scopo, in periodi diversi, furono posti, agli estremi del lago (Marta e Bolsena), due limnografi, espressamente costruiti. Difficoltà varie, nonché l'opportunità di dedicare la mia attenzione ad altre ricerche, rivelatesi di più urgente intervento, hanno fatto sì che le osservazioni in parola siano state, finora, solo saltuarie e intervallate da lunghi periodi di sosta. Esse, pertanto, continueranno.

Le serie già ottenute presentano però - a mio avviso - un interesse particolare, avendo rivelato l'esistenza di un insospettato fenomeno che giuoca un ruolo rilevante nell'alternarsi delle oscillazioni del lago di Bolsena. Scopo di questa nota è appunto quello di dar notizia - e ragione nello stesso tempo - di detto fenomeno.

\section{3. - LE OSSERVAZIONI.}

1958-1959 - Come si è detto, le osservazioni clinografiche sul lago di Bolsena non hanno finora assunto carattere sistematico.

Per quanto riguarda il 1958, esse si limitano agli ultimi giorni dell'anno. Si era cominciato imponendo allo strumento, funzionante a Marta, nella parte meridionale del lago, un ingrandimento dell'ordine di 2. Ci si rese subito conto dell'inopportunità di tale ingrandimento. Normalmente, infatti, nel lago di Bolsena le oscillazioni libere, specie a Marta, raggiungono ampiezze apprezzabili, spesso cospicue, a differenza 
degli altri laghi dell'Italia centrale, quello di Albano compreso. Per evitare la fuoruscita della pennina, si è portato l'apparecchio a registrare le oscillazioni in grandezza naturale.

La Fig. 1 riporta alcuni tratti di registrazione, ottenuti con l'apparecchio raddoppiante le ampiezze.

La Fig. 2 riproduce tratti di registrazione, relativi ai giorni 1-2 del Gennaio 1959: l'ingrandimento è $1: 1$.

Tratti di registrazione ottenuti il 25-II-1959 sono nella Fig. 3, mentre le Figg. 4 e 5 riportano chiari esempi di uninodale (che è nettamente l'oscillazione predominante, se non esclusiva del lago) ottenuti dal 23 al 26 Febbraio 1959.

1962-1963. - Lo scorrimento del limnografo, nelle indagini del 1962 e del 1963, fu tenuto più piccolo: constatato che l'oscillazione quasi esclusiva del lago era quella uninodale, si è ritenuto superfluo insistere con gli scorrimenti del periodo precedente.

La Fig. 6 riporta le registrazioni ottenute a Marta dal 6 al 12 Febbraio 1962. La sequenza dei battimenti è pressoché sempre presente, non ostante essa venga spesso alterata dall'insorgere improvviso della causa perturbante, specie nelle ore in cui soffia il vento (rivelato dalla presenza di "shelf-seiches", che orlano la registrazione delle uninodali).

Esempi di registrazioni ottenute nel 1963 sono quelli riportati nelle Figg. 8, 9, 10, 11.

Di particolare interesse risultano quelle registrazioni nelle quali l'uninodale appare unica, nel senso che lungo una sola sezione del lago si verifica l'alternarsi dell'oscillazione stazionaria: tali casi sono caratterizzati da persistenza dell'oscillazione, la cui ampiezza decresce gradualmente, secondo il naturale smorzamento. In questi casi, in cui mancano i battimenti, il periodo è quasi sempre il massimo consentito (da $14^{\mathrm{m}}$ a $15^{\mathrm{m}}$ ), a significare che, quella che talvolta appare singolarmente, è l'uninodale fondamentale del lago (Figg. 5, 7).

4. - Si osservino le registrazioni riportate. Balza subito all'occhio che esse generalmente si presentano come lunghe serie di battimenti (mi riferisco alle uninodali): v. Figg. 12 e 13.

Era pertanto da ritenere che, quello tratto come media dalle registrazioni stesse, andasse considerato come " pseudo-periodo " e non come periodo dell'oscillazione fondamentale, la quale doveva consistere, in 
realtà, dall'interferenza di due oscillazioni contemporanee, aventi periodo ad ampiezze poco diversi tra loro.

Siano $T_{1}$ e $T_{2}\left(\operatorname{con} T_{2}>T_{1}\right)$ i periodi delle due uninodali. di ampiezza pressoché ugruale, destate nel lago di Bolsena. Indicando con $v$ la velocità di propagazione, possiamo serivere:

$$
u_{1}=A \sin \frac{2 \pi}{\tilde{l}_{1} v}(t v-x), \quad u_{2}=A \sin \frac{2 \pi}{\pi_{2} v}(t v-x),
$$

sicché, la loro composizione, $u=u_{1}+u_{2}$, risulta di equazione:

$$
u=2 A \cos \frac{2 \pi}{v}(t v-x) \frac{T_{2}-T_{1}}{2 T_{1} T_{2}} \cdot \sin \frac{2 \pi}{v}(t v-x) \frac{T_{1}+T_{2}}{2 T_{1} T_{2}}
$$

Si tratta ancora di un'onda sinusoidale, la cui ampiezza

$$
2 A \cos \left\{\frac{2 \pi}{v}(t v-x) \cdot \frac{T_{2}-T_{1}}{2 T_{1} T_{2}}\right\}
$$

risulta variabile nel tempo.

Fatto $T_{2}=T+\varepsilon, T_{1}=T-\varepsilon$, essendo $\varepsilon$ una quantitì infinitesima, potremo scrivere:

$$
u=2 A \cos \frac{2 \pi}{\lambda}(t v-x) \frac{\varepsilon}{T} \cdot \sin \frac{2 \pi}{\lambda}(t v-x),
$$

essendo $\lambda=T v$. Sarà questa la risultante delle due oscillazioni di periodo $T_{1}$ e $T_{2}$.

Riprendiamo la [1]. Indichiamo con $p$ e $P$ rispettivamente il periodo dell'oscillazione risultante e il periodo di ogni battimento $(P / 2$ esprime quindi l'intervallo in cui insorge e svanisce un battimento).

Avremo

$$
\frac{2}{p}-\frac{T_{1}+T_{2}}{T_{1} \cdot T_{2}}, \quad \frac{2}{P}=\frac{T_{2}-T_{1}}{T_{1} \cdot T_{2}}
$$

Da cui

$$
\frac{P+p}{p P}=\frac{1}{T_{1}} ; \quad ; \quad \frac{P-p}{p P}=\frac{1}{T_{2}}
$$

e quindi

$$
T_{1}=\frac{p P}{p+P} ; \quad T_{\mathrm{2}}=\frac{\tilde{P}^{D}}{P-p}
$$


Conosciuti $p$ e $P$, le [2] ci danno subito $T_{1}$ e $T_{2}$.

Dalle oscillazioni si deduce, in media, $p=12^{\mathrm{m}} \cdot 6, P=135^{\mathrm{m}}$. Ne consegue, per le due oscillazioni componenti,

$$
T_{1}=11^{\mathrm{m}} .5, \quad T_{2}=13^{\mathrm{m}} \cdot 9 .
$$

Il periodo medio $T=12^{\mathrm{m}} .6$ - che coincide praticamente con $p-$ delle registrazioni ottenute a Marta e a Bolsena, non è quindi il periodo dell'uninodale, che - per detto lago - non è unica. Esso è il periodo medio degli elementi dei battimenti, risultanti dalla composizione di due oscillazioni uninodali, oscillanti sul ritmo di $11^{\mathrm{m}} . \dot{b}$ e $13^{\mathrm{m}} .9$ rispettivamente.

Si hanno però altre combinazioni di valori per $p$ e $P$. Per esempio, $p=12^{\mathrm{m}} .9, P=84^{\mathrm{m}}$, dai quali si ottengono

$$
T_{1}=11^{\mathrm{m}} .2, \quad T_{2}=15^{\mathrm{m}} .2 ;
$$

oppure, $p=12^{\mathrm{m}} .9, \quad P=102^{\mathrm{m}}$, da cui si deduce

$$
T_{1}=11^{\mathrm{m}} \cdot 45, \quad T_{\mathrm{a}}=14^{\mathrm{m}} \cdot 8 \text {. }
$$

Queste diverse combinazioni devono essere attribuite a diverse sezioni longitudinali del lago, lungo le quali l'acqua compie l'oscillazione fondamentale.

In altre combinazioni (Marta, tra le $19^{\mathrm{h} 20^{\mathrm{m}}}$ e le $20^{\mathrm{h} 25^{\mathrm{m}}}$ del I-I-1959) risulta $p=13^{\mathrm{m} .0}$ e $P=103^{\mathrm{m}}$, da cui

$$
T_{1}=11^{\mathrm{m}} .5 \pm ; \quad T_{2}=14^{\mathrm{m}} .88 \text {. }
$$

Inoltre (intorno alle $14^{\mathrm{h}}$ del $25-\mathrm{II}-1959$ ), si ottiene $p=13^{\mathrm{m}}, P=87^{\mathrm{m}}$ e quindi

$$
T_{1}=11^{\mathrm{m}} .3 ; \quad T_{2}=15^{\mathrm{m}} .23 .
$$

E questa una caratteristica che si manifesta, in modo netto, solo per il lago di Bolsena (almeno fra i laghi finora indagati). ì mio proposito chiarire il mecanismo di effettuazione di detto fenomeno.

5. - Dalle oscillazioni registrate contemporaneamente a Marta e a Bolsena è anche possibile trarre il rapporto delle ampiezze, con le quali le uninodali si manifestano ai due estremi, meridionali e settentrionali, del lago. Si veda il confronto di un tratto di registrazione contemporanea di uninodali, ottenute nelle due località citate il 1963 , e riportate nella Fig. 11. 
Da esso si trae agevolmente che le uninodali registrate a Marta hanno un'ampiezza circa 3 volte maggiore dell'ampiezza osservata a Bolsena. Ma questo rapporto non è conservato costante. Sovente specie per gli spostamenti di piccola ampiezza - le uninodali a Marta sono solo 2,5 volte più ampie che a Bolsena; talvolta a Marta possono risultare di ampiezza quadrupla di quella osservata a Bolsena. È da ritenere ciò debba ascriversi al fatto che, i passaggi dei disturbi microbarici - ai quali, sopra tutto, sono dovute le perturbazioni dei laghi avvengano in modo da esaltare, ora ad un estremo ora ad un altro, il movimento ivi in atto; o viceversa. In ogni caso, il rapporto delle ampiezze fra Marta e Bolsena ̀̀, in media, nettamente superiore di quello previsto dal calcolo $\left({ }^{3}, 4\right)$.

6. - Dò ora notizia di un'altra esperienza sulle rive del lago di Bolsena, a Marta.

Ho già detto dell'abituale, notevole ampiezza raggiunta dalle oscillazioni libere uninodali nella parte Sud del lago. Ho pensato che, la differenza di carico, determinata sul fondo del lago, presso la riva, doveva essere capace di determinare una variazione della verticale apparente, registrabile con un clinometro, sufficientemente sensibile.

A questo scopo, in un locale presso la riva del lago, fu posto in funzione, per qualche giorno, un clinografo avente un periodo proprio di $22 \sec$ ca $\left(^{5}\right)$.

L'esperienza ebbe esito positivo. Infatti, in occasione di sesse di media ampiezza, il clinografo registra chiare variazioni della verticale apparente, coperiodali alle sesse stesse (Figg. 14, 15, 16). Tali registrazioni possono ritenersi delle vere e proprie sesse so?ille: alle estremità del lago, hanno quindi origine, in corrispondenza dell'alternarsi delle oscillazioni libere, delle onde progressive che si propagano nel mezzo solido - con periodo uguale a quello delle onde liquide e con ampiezza rapidamente decrescente - verso Sud e verso Nord, rispettivamente (Fig. 17).

$\Delta d$ un'uninodale di ampiezza di $11 \mathrm{~cm}$ ca, corrisponde un'ondulazione solida di 0.3 sec d'arco ca. di ampiezza.

Le costanti del limnografo, durante la registrazione delle "sesse solide ", erano tali da tradurre la variazione di un secondo d'arco nello spostamento di mm 15 dell'imagine luminosa. Poiché oscillazioni libere di un'ampiezza di $\mathrm{cm} 6$ ca. provocano (v. Fig. 16) spostamenti pari a mm 2.5 ca., se ne deduce che tali spostamenti corrispondevano a variazioni della verticale apparente pari a 0.17 secondi d'arco. 
P. Caloi
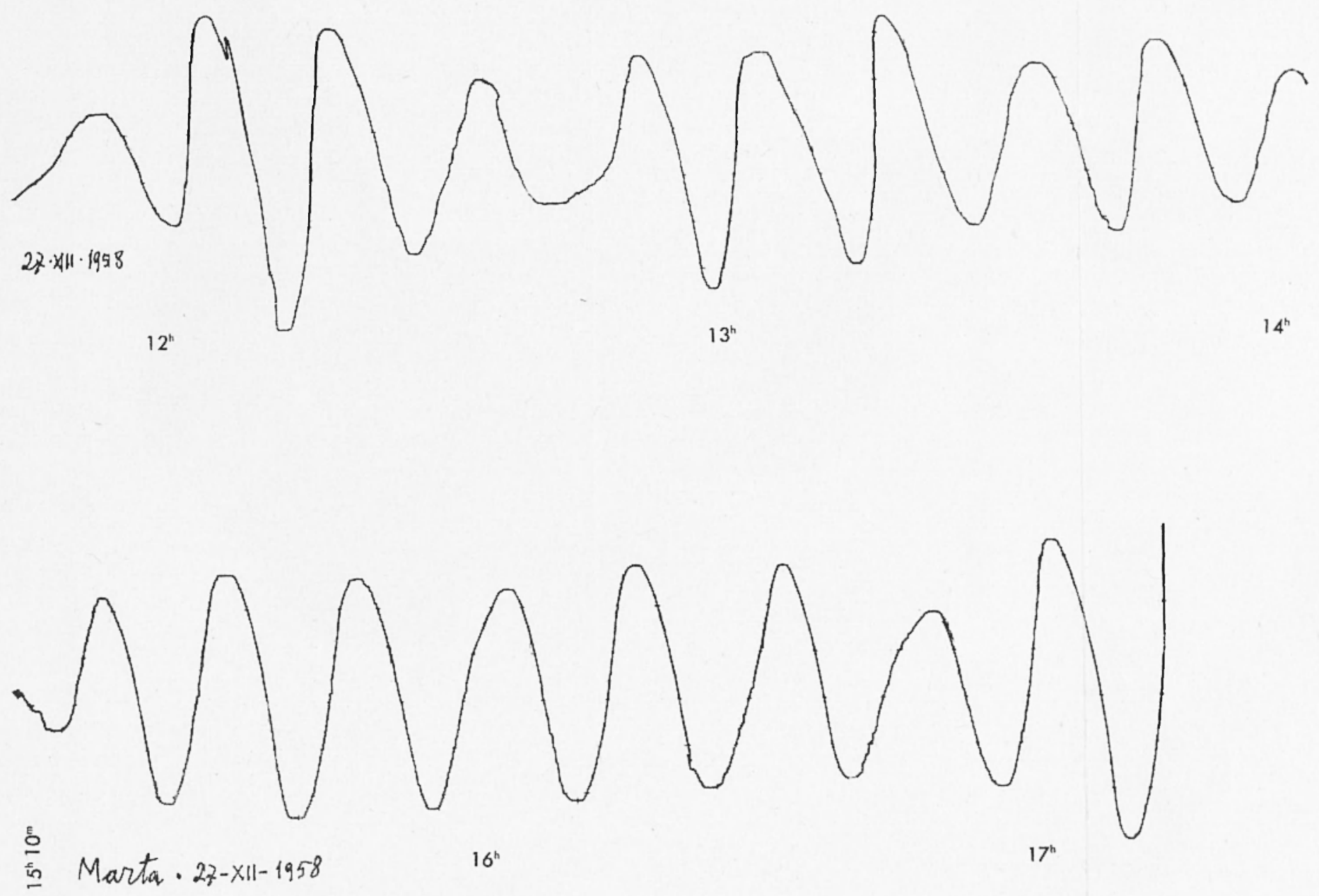

Fig. 1 - Esempi di uninodale a Marta (ampiezza 2/3 dell'originale). 


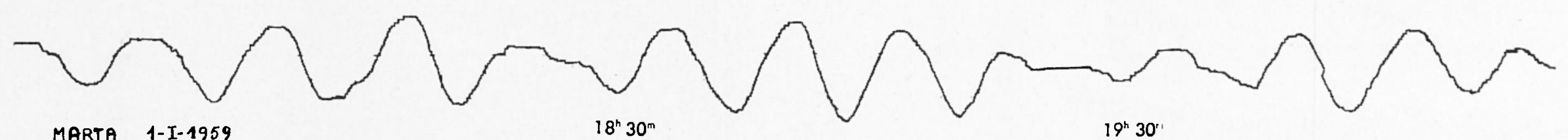

MARTA $1-\mathrm{T}-1959$

$18^{\mathrm{h}} 30^{\mathrm{m}}$

$19^{h} 30^{\prime}$

Fig. 2 - Uninodali in successione di battimenti (riduzione $2 / 3$ dell'originale).
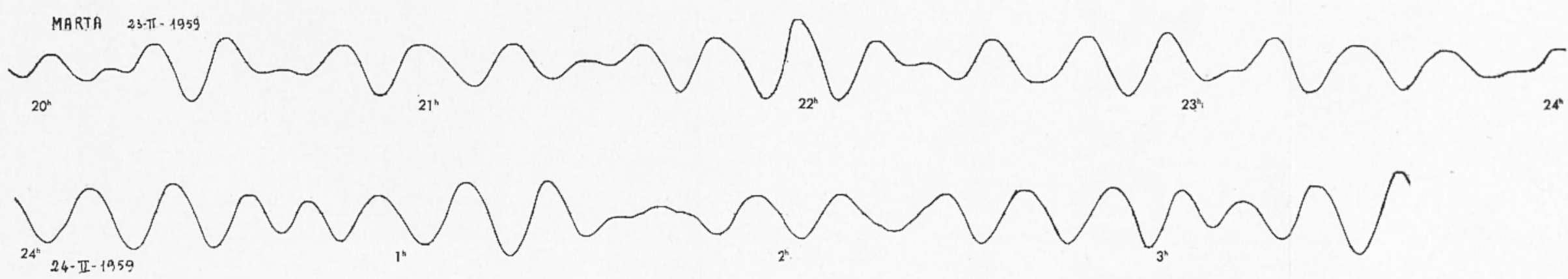

1. MARTA
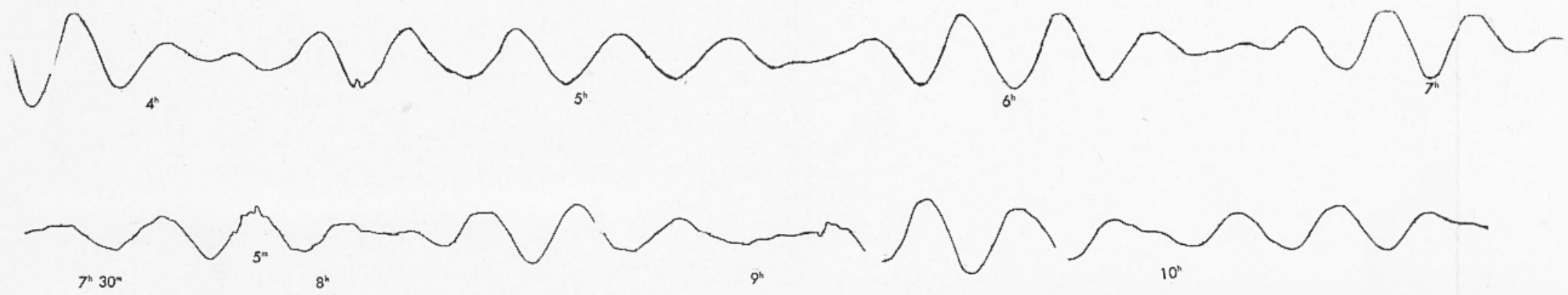

Fig. 3 - I battimenti delle uninodali vengono sovente alterati da improvvise insorgenze di nuove fondamentali o da variazioni delle ampiezze delle oscillazioni in ginoco (riduzione a $1 / 2$ dell'originale). 

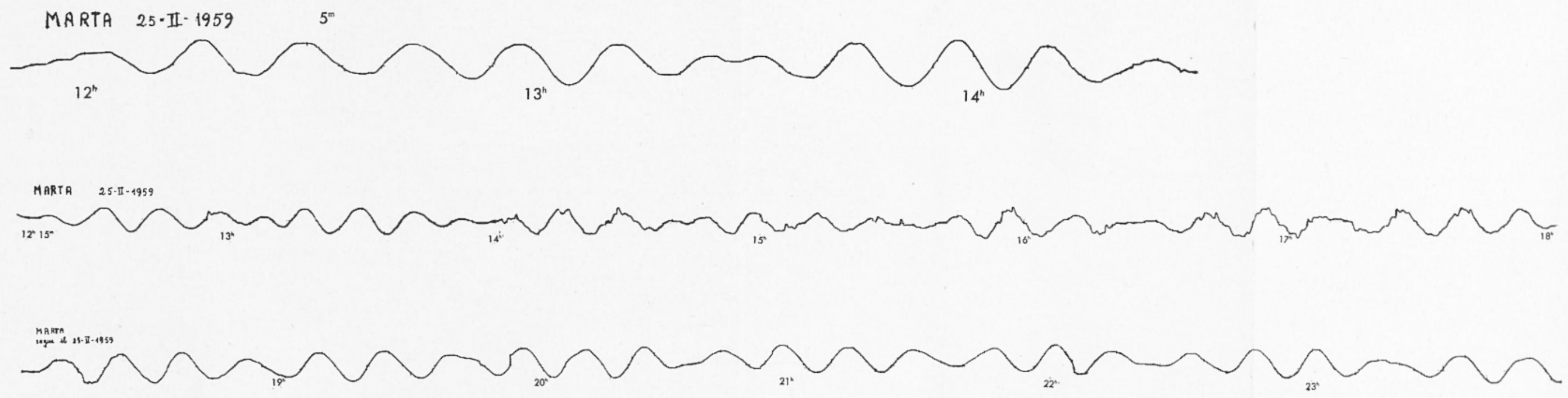

Fig. 4 - Verso le $14^{\mathrm{h}}$ del $25.1 \mathrm{II} .59$ i battimenti riprendono il normale andamento (ampiezza $2 / 3$ dell'originale).
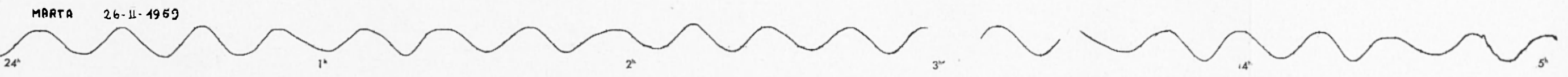

Fig. 5 - Serie di battimenti per uninodali inizialmente di ampiezza pressoché uguale; lentamente, poi, una delle uninodali si attenua, fino a scomparire (riduzione a ca. $1 / 2$ dell'originale). 
P. Carot
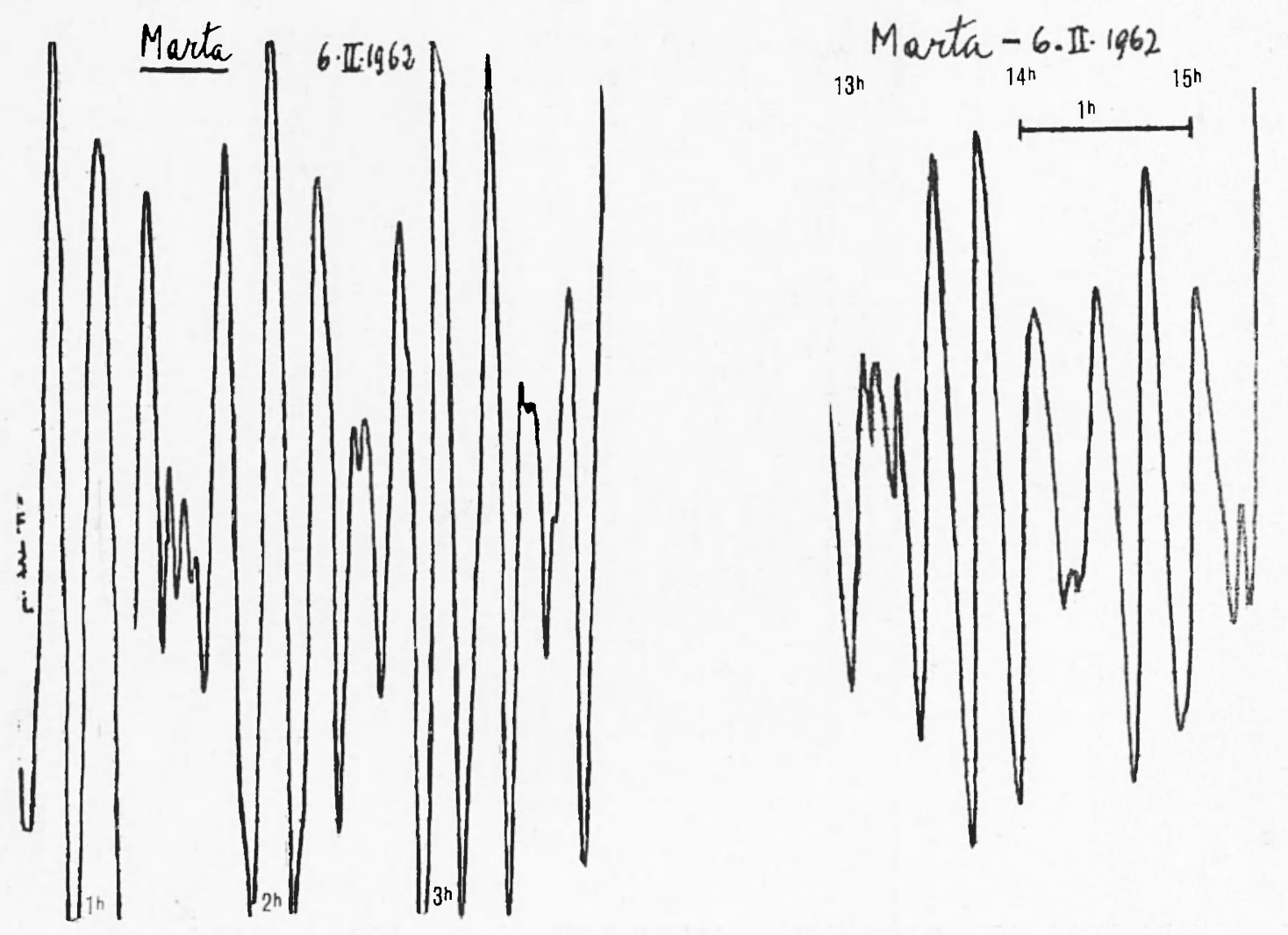

Marta. $6 \cdot \pi \cdot 1962$

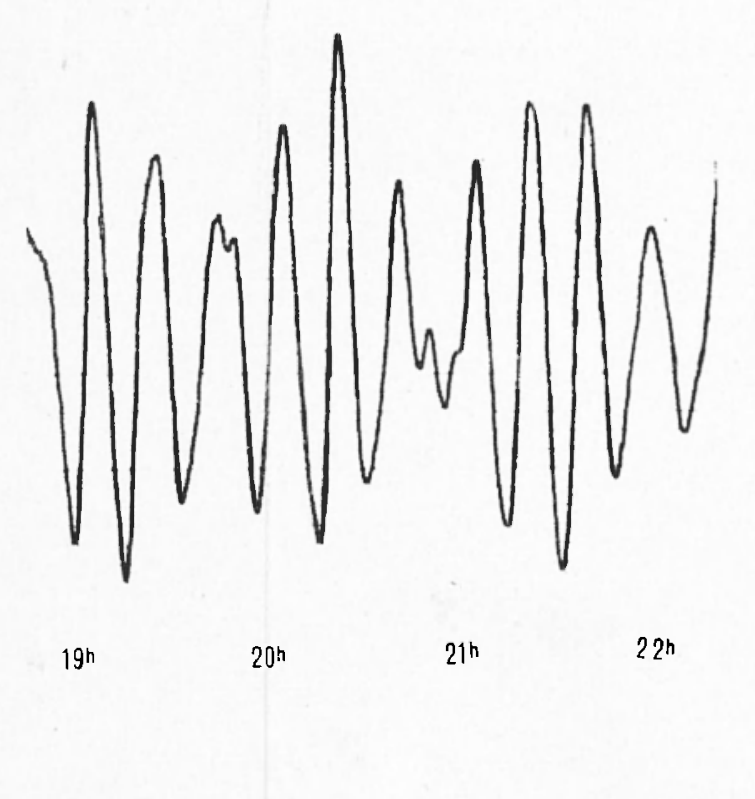

MARTA $12-$ II -1962

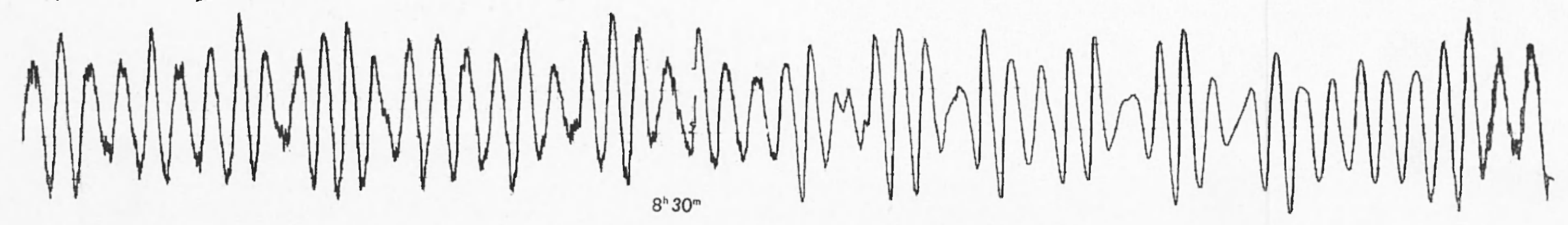

Fig. 6 - Lunga serie di ampie uninodali, registrate a Marta, con l'abituale composizione a battimenti. Il moto libero del lago si attenua lentamente, alterando, di quando in quando, la snccessione dei battimenti. 

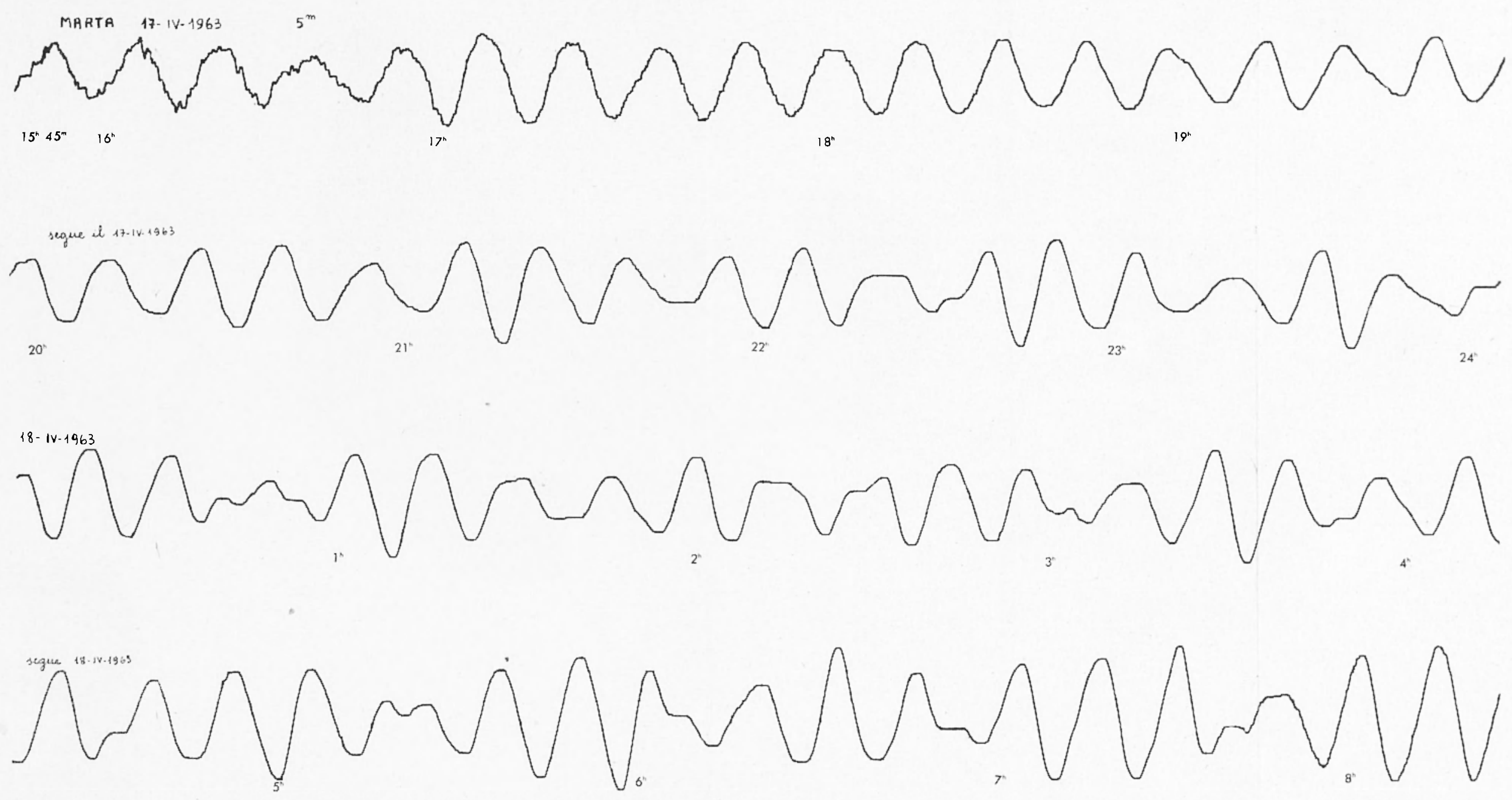

Fir. 7 - Dopo periodi di prevalenza di una delle due fondamentali lentamente i battimenti tornano a riformarsi (riduzione $1 / 2$ ). 
P. CALOI

BOLSENA

$28-x 11-1963$

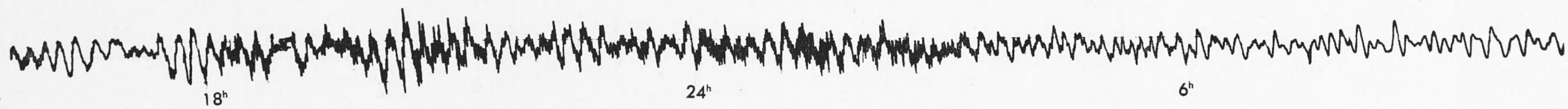

BOLSENA $26-x 11-1963$

$27-x 11-1963$

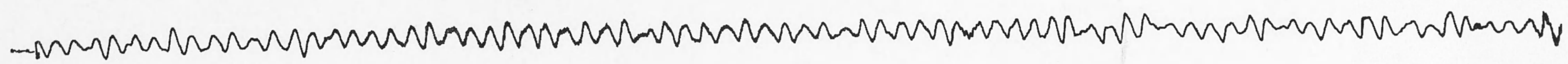

b) $20^{\mathrm{h}} 30^{\mathrm{r}}$

$24^{\mathrm{h}}$

$6^{\mathrm{h}}$

$12^{\mathrm{h}}$

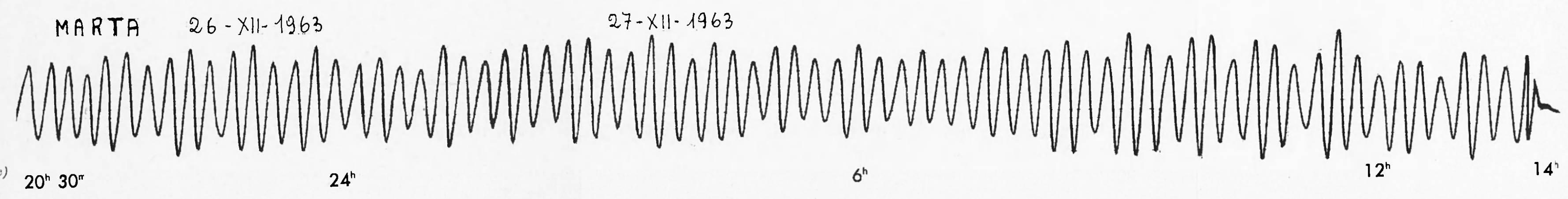

Fig. 8 - In a) l'uninodale (registrata a Bolsena) appare orlata di vibrazioni a breve periodo, dovute a vento ("slielf-seiches"). In $b$ ) e in $c$ ) si hanno due tratti di contemporanee registrazioni a Bolsena e a Marta, ai lati opposti del lago: trattandosi di uninodali, le singole os
mente in opposizione di fase. A Marta le ampiezze sono circa quadruple di quelle ottenute contemporaneamente a Bolsena. 


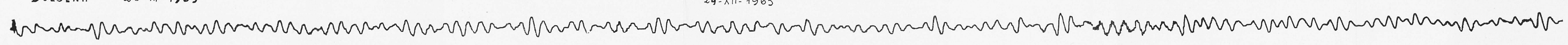

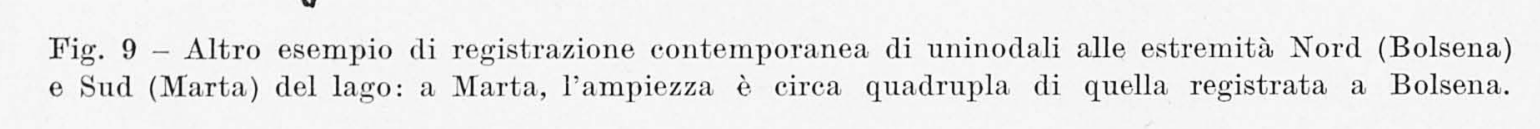

\section{BOLSENA 29-X11-1963}

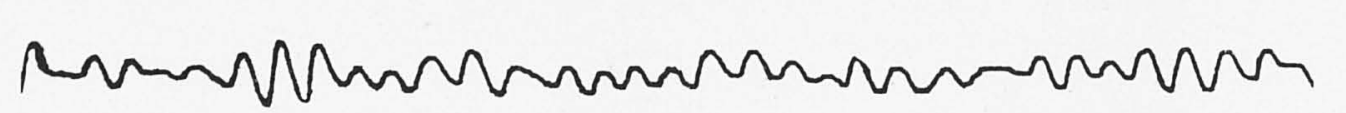

MARTA 29-X11-1963

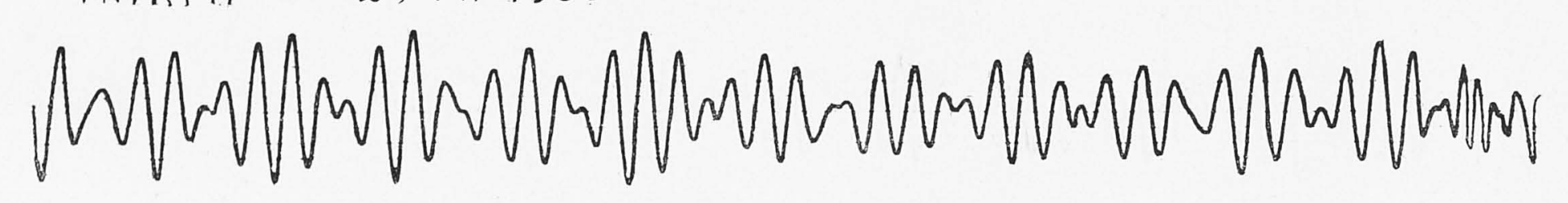

Fig. 10 - Altro sempio di registrazione contemporanea di uninodali alle estremitia Nord (Bolsena)
o Sud (Marta) del lago: a Marta, lampiezza e circa quadrupla di quella registrata a Bolsena.

MARTA $30-\times 11=1963$

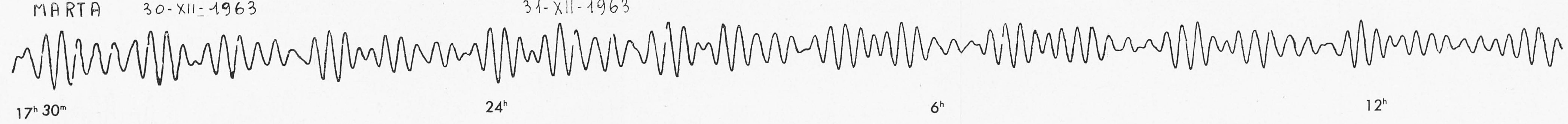

BOLSENA 30-x11.1963

$31-x 11-1963$

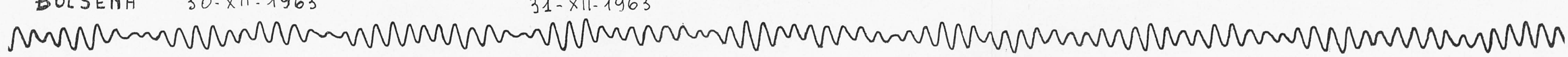




$$
\text { HARTA }
$$




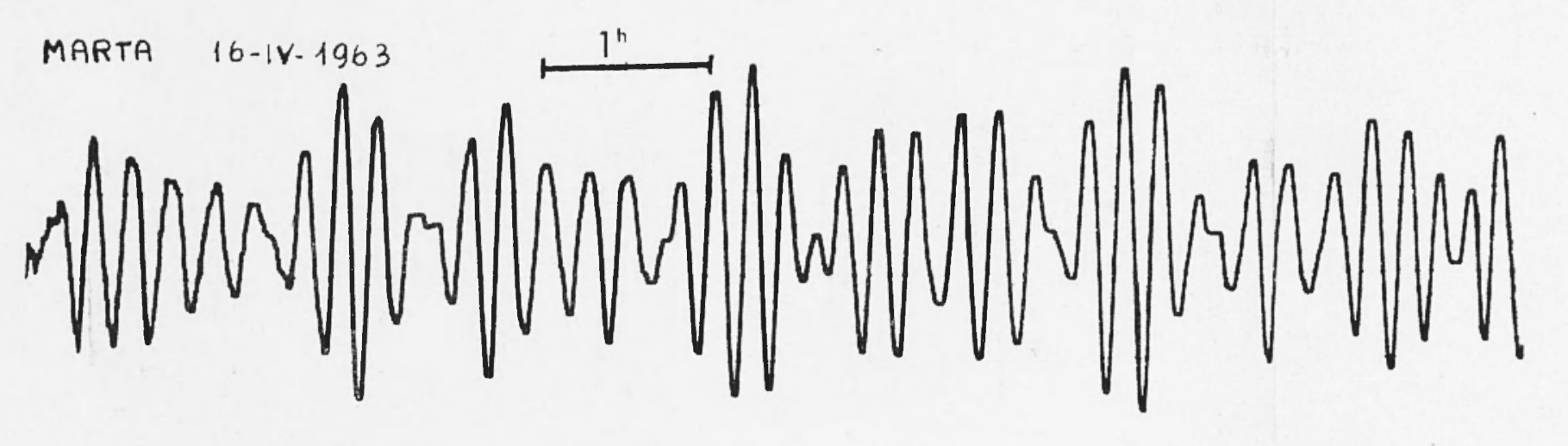

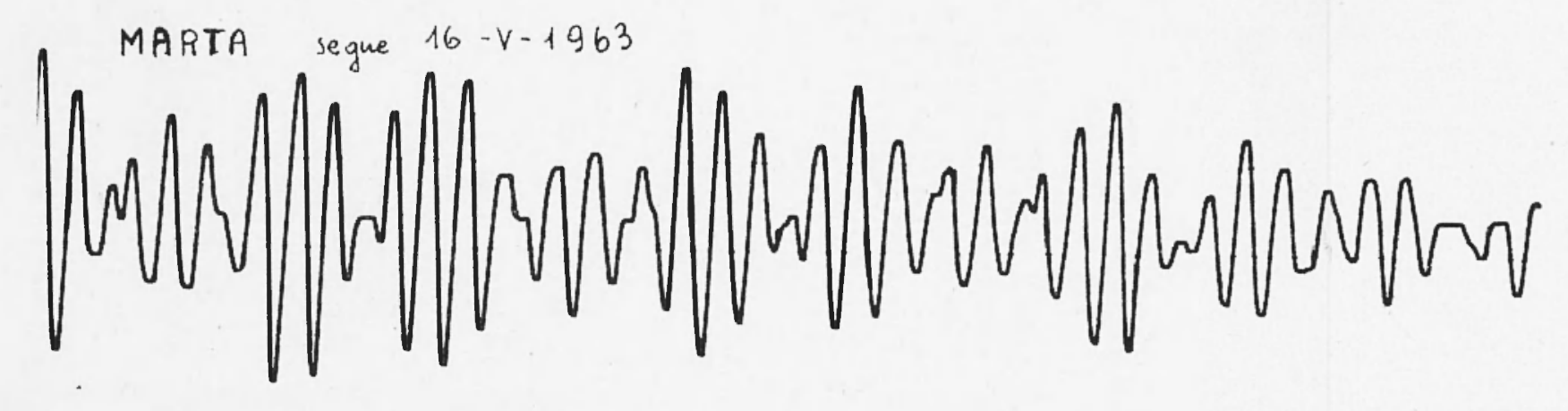




\section{MARTA $22-1 V-1963$}

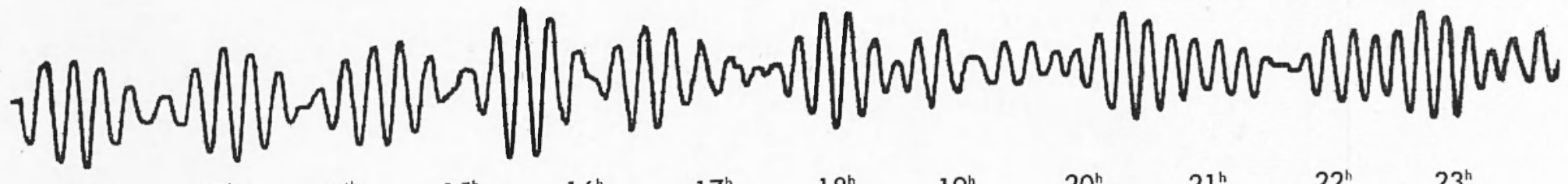
$12^{\mathrm{h}}$ $13^{\mathrm{h}}$

$14^{\mathrm{h}} \quad 15^{\mathrm{h}}$

$16^{\mathrm{h}}$

$17^{\mathrm{h}}$

$18^{\mathrm{h}} \quad 19^{\mathrm{h}}$

$20^{\mathrm{h}} \quad 21^{\mathrm{h}}$

22

$23^{\mathrm{h}}$

$23-1 V-1963$

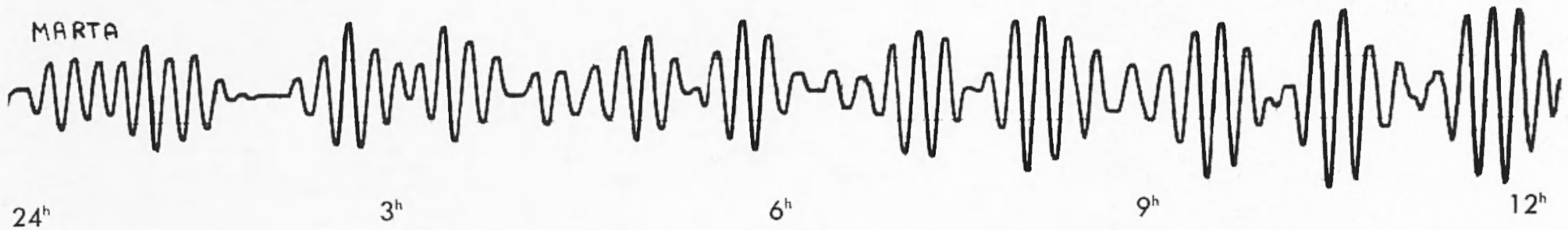

MARTA Segue 23-1V-1963

$24-I V-1963$

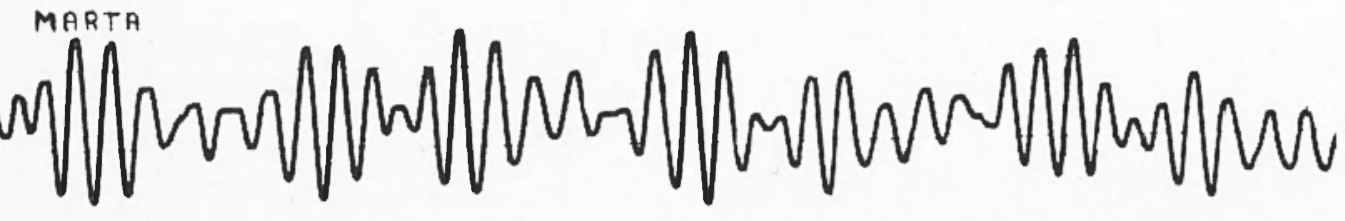

$24^{h}$

$6^{\mathrm{h}}$

Fig. 14 - In queste registrazioni, l'oscillazione su due diverse sezioni longitudinali, dura, a Iungo, immutata. 
La Fig. 18 rappresenta in forma schematica, l'andamento dell'uninodale ai due estremi del lago e il trasferimento, in detti estremi, dell'energia in sessa solida.

7. - Sulla base delle registrazioni ottenute, possiamo valutare l'ordine di grandezza dell'energia cinetica di un'oscillazione libera uninodale.

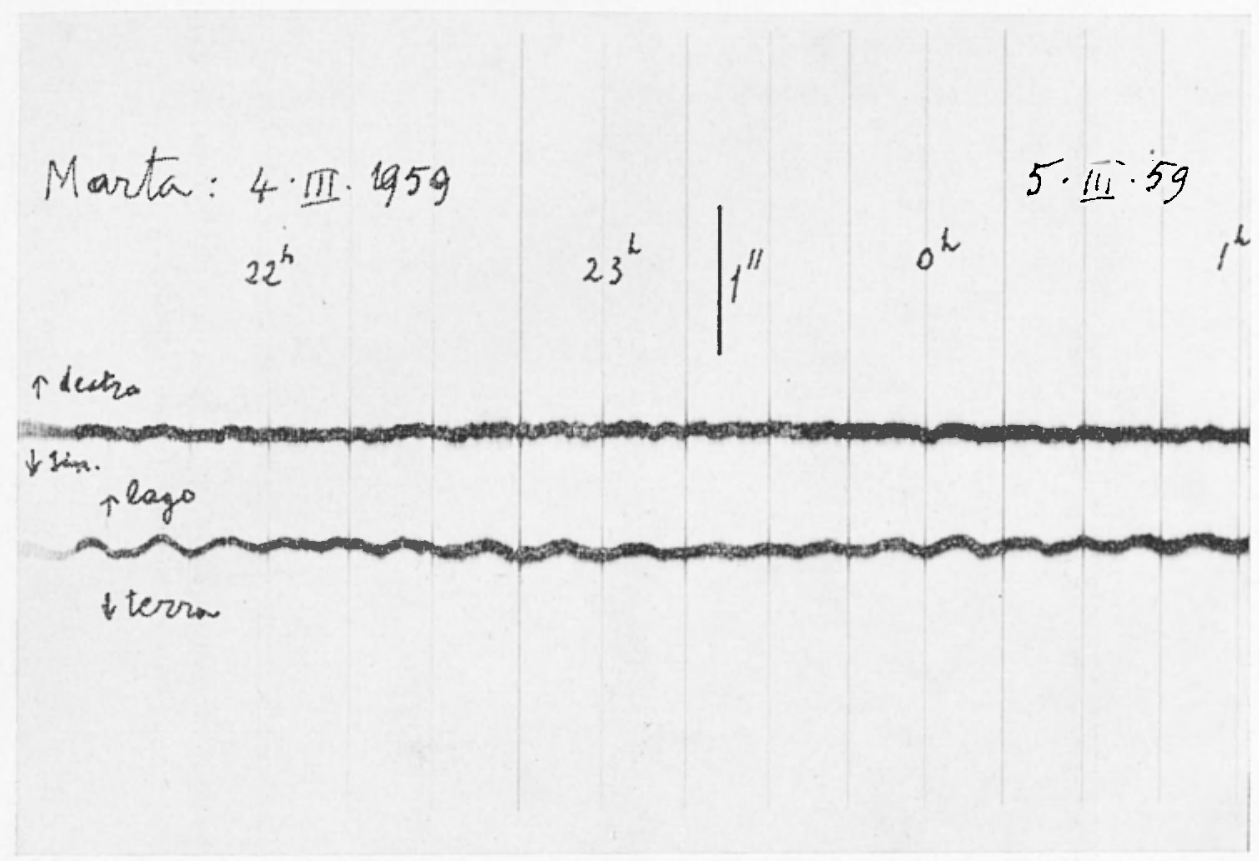

Fig. 15 - Ie aninodali, a Marta, trasferiscono parte dell'energia al suolo, causanclo la registrazione di coperioclali variazioni della verticale apparente (sesse solide).

E noto che, equiparando il bacino di un lago ad un parallelepipedo di lunghezza $l$, larghezza $b$ e profondità $h$, l'energia cinetica $E_{c}$, animante un'oscillazione libera uninodale di periodo $T_{o}$, vale $\left(^{\circ}\right)$

$$
E_{c}=\frac{1}{4} \varrho b h l C^{2} \sin ^{2} \frac{2 \pi}{\Pi_{o}} t
$$




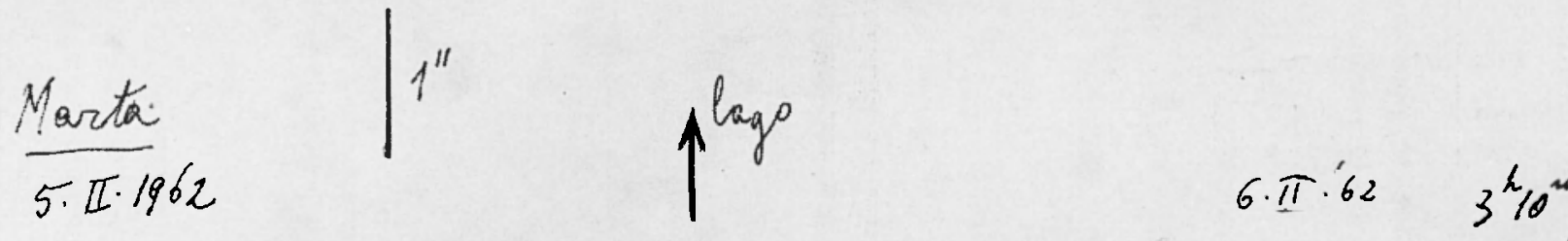

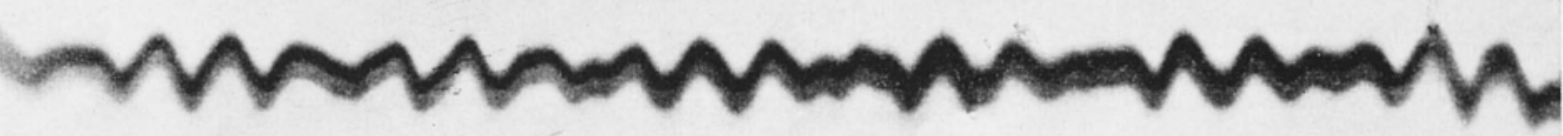
$120^{x} 20^{m}$ $i_{\text {tenna }}$

EFig. 16 - Altro notevole esempio di sesse solide, registrato dal clinografo di Marta, in_corrispondenza di coperiodali (sesse liquide). 


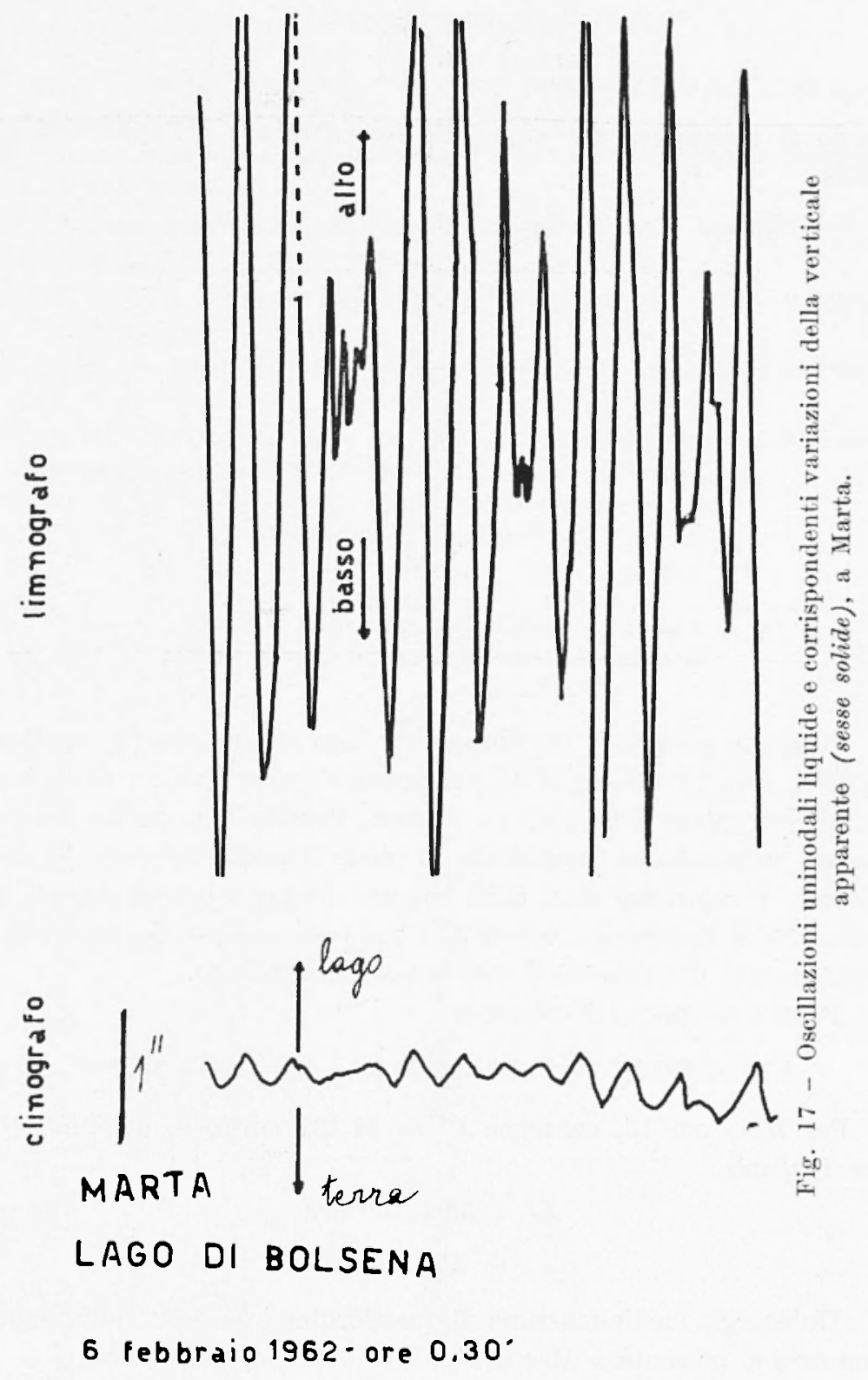


dove $\varrho$ è la densità dell'uequa e

$$
C=H \sqrt{\frac{g}{h}},
$$

essendo $H$ l'ampiezza dell'uninodale considerata e $g$ l'accelerazione di gravità.

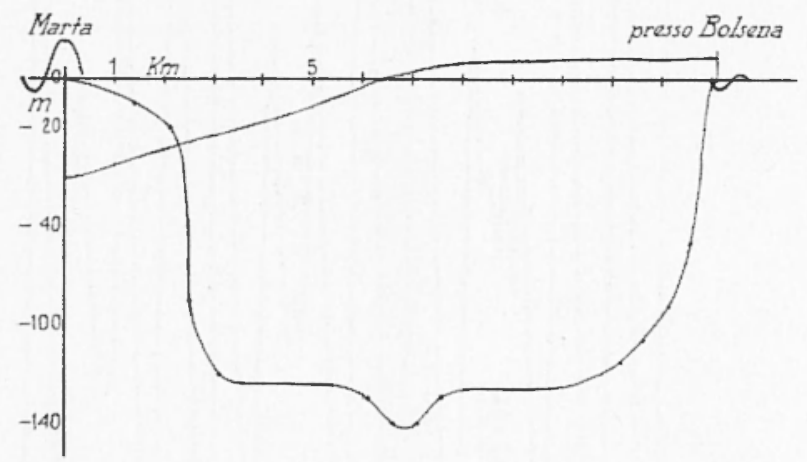

Fig. 1s - Oscillazioni uninodali a Marta e

a Bolsena, nolle loro fasi liguida e solida.

Il primo lavoro di Di Filippo sul lago di Bolsena $\left({ }^{3}\right)$, contiene a pag. 276, i dati morfologici da eni possono trarsi i valori di $l, b$ e $h$ : $l$ è l'ultimo valore della colonna $2^{a}$ della Tabella I; la media dei valori

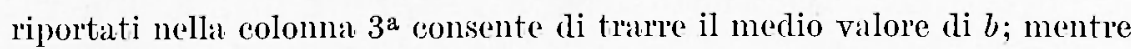
dividendo $\mathrm{i}$ valori riportati nella colomna $6^{\mathrm{a}}$ per $\mathrm{i}$ corrispondenti della colonna $3^{\mathrm{a}}$, si deducono i valori di $h$ per ogni sezione, donde segue immediatamente il valore di $h$ medio per l'intero lago.

Fatti i calcoli, si è ottenuto

$$
b=\mathrm{m} \mathrm{8254,} \quad h=\mathrm{m} 70,9, \quad l=\mathrm{m} 13.000 .
$$

Per $H=\mathrm{cm} \mathrm{10}$, consegne $C^{-}=31,132 \mathrm{~cm}^{2} / \mathrm{sec}^{2}$, e quindi (fatto $\left.\underline{o}=1 \mathrm{gr} / \mathrm{cm}^{3}\right)$,

$$
\begin{aligned}
E_{c} & =\tilde{5}, 92 \cdot 10^{26} \text { erg } \\
& =1640 \mathrm{kwh} .
\end{aligned}
$$

Un'energia medio-massima di quest'ordine è associata all'uninodale, come essa si presenta a Marta. Sul lato opposto, a Bolsena, ci si deve attendere un'energia $1 / 16$ di quella su riportata, in quanto - generalmente - l'uninodale manifesta a Bolsena ampiezze circa 4 volte minori di quelle osservate a Marta. 
CARATTERISTICHE DELLE OSCILLAZIONI LIBERE DEL LAGO DI BOLSENA 305

\section{BIBLIOGRAFIA}

(1) Palazzo I., Studi limnografici sul lago di Bolsena. "Atti del Vo Congresso Geografico Italiano », 2, Sez. I, (1940).

(2) P. Caloi, Le sesse del lago di Garda. Parte I e II, "Anuali di Geofisica", I, 1 e 2, (1948).

(3) D. Dr Filippo, Le sesse del lago di Bolsena, "Annali di Geofisica ", IV, 2, (1951).

(') D. Di Fil.mpo, Ulleriore studio sulle sesse del lago di Bolsena, "Annali di Geofisica ", VI, 3, (1953).

(5) P. Calor, Il pendolo orizzontale come clinometro, "Annali di Geofisica", III, 4, (1950).

$\left({ }^{6}\right)$ P. Calor, Oscillazioni libere del lago di Bracciano: loro caratteristiche e loro energia. "Annali di Geofisica ", XI, 1-14, (1958). 